\title{
PROGRAMAÇÃO DA PRODUÇÃO HÍBRIDA PARA MINIMIZAR O CUSTO TOTAL DO CONSUMO DE ENERGIA
}

\author{
Carlos Guilherme Souza Koehler (Instituto Federal do Espírito Santo - IFES) \\ carlos.cgsk@gmail.com \\ Mário Mestria (Instituto Federal do Espírito Santo - IFES) \\ mmestria@ifes.edu.br; mmestria@uol.com.br
}

\section{Resumo}

Problemas de programação da produção em um ambiente de produção híbrida são bastante pesquisados, entretanto o foco principal das pesquisas tem sido a eficiência da produção, pouca atenção tem sido dada ao consumo de eletricidade. Esse trabalho tem como foco utilizar um algoritmo genético para considerar além do tempo total de produção o consumo de energia elétrica. Os resultados desse trabalho são comparados com resultados de referência da literatura e foram geradas 20 instâncias considerando o consumo de energia elétrica onde foi avaliado o impacto da estratégia multiobjetivo no tempo de produção. Os resultados dessa pesquisa têm significantes implicações na gestão da produção de sistemas reais.

Palavras-Chaves: Otimização, consumo de energia, programação da produção híbrida, otimização multiobjetivo, algoritmo genético.

\section{Introdução}

O foco deste trabalho são os problemas da programação da produção (Job Shop Scheduling Problem), um dos problemas da classe da programação das operações. Esta classe de problemas, segundo Pinedo (2016), é uma das mais críticas no planejamento e gerenciamento de processos de manufatura, sendo a parcela do PIB relativa a este setor no Brasil igual a $12 \%$ (WORLDBANK, 2016). Pezzella, Morganti e Ciaschetti (2008) consideram a programação da produção, como um dos problemas da programação das operações mais difíceis. No problema clássico da programação da produção um conjunto de trabalhos deve ser processado por um conjunto de máquinas, cada trabalho é formado por uma sequência de operações consecutivas, cada operação requer exatamente uma máquina e máquinas estão disponíveis e são capazes de processar uma operação por vez sem interrupções, este processo de decisão consiste em determinar a sequência de processamento das operações nas máquinas. 
Dentre os problemas da programação da produção destaca-se o problema da produção híbrida (hybrid flow shop scheduling), onde são introduzidas máquinas em paralelo em cada estágio da produção. De acordo com Ruiz e Vázquez-Rodríguez (2010) e Fan et al. (2018) o problema da produção híbrida é um problema amplamente encontrado na indústria da manufatura. Segundo Luo et al. (2013) os trabalhos envolvendo configurações de produção híbridas têm como foco principal a eficiência da produção, minimizando o tempo total de produção, o atraso total e outros aspectos referentes a eficiência da produção. Entretanto esses não são os únicos aspectos que deveriam ser considerados., também precisam ser considerados os aspectos ambientais, e um deles é o consumo de energia elétrica, principalmente levando em conta que o setor industrial foi responsável por $35 \%$ do consumo de energia no Brasil em 2017 (EPE, 2017).

A introdução de máquinas em paralelo em cada estágio da produção torna o problema da produção hibrida mais difícil do que a programação da produção clássica pois introduz mais um nível de decisão além do sequenciamento. Nesse sentido programação da produção híbrida é NP-Difícil, o que significa que não são conhecidos algoritmos exatos para sua solução em tempo computacional aceitável (CORMEN et al., 2009). Conforme demonstrado por Gupta (1988) numa programação da produção híbrida com dois estágios, mesmo com um estágio com uma máquina e no outro com duas o problema é NP-Difícil.

Neste trabalho, os critérios explorados na resolução do problema da produção hibrida são o tempo total de produção e o consumo de energia elétrica, classificando o problema como multiobjetivo. O trabalho busca desenvolver um algoritmo genético para obter as soluções para o problema, sendo utilizado as instâncias propostas em Hurink, Jurisch e Thole (1994), de forma a validar a parte inicial do trabalho. Então o algoritmo é avaliado utilizando novas instâncias que incluem o consumo de energia elétrica das máquinas com o objetivo fazer uma comparação do impacto da avaliação do consumo de energia elétrica no tempo total de produção.

O artigo é estruturado da seguinte forma: na segunda seção é descrito o problema e suas formulações e na terceira seção uma breve revisão bibliográfica é realizada. Na quarta seção é apresenta a metodologia e na quinta seção os resultados e as discussões. Na última seção são descritas as considerações finais. 


\section{Definição do problema}

O problema da programação produção híbrida (HFS), é um problema comum da manufatura que apresenta várias variações, e todas as variações devem ter as seguintes características em comum (RUIZ; VÁZQUEZ-RODRIGUEZ, 2010).

- O número de estágios de processamento m deve ser de pelo menos 2;

- Cada estágio $\mathrm{k}$ tem pelo menos uma máquina em paralelo $(\mathrm{Mk}>=1)$ e em pelo menos um dos estágios o número de máquinas em paralelo é maior do que $1(\mathrm{Mk}>1)$;

- Todos os trabalhos devem ser processados na mesma ordem de produção: estágio 1, estágio $2, \ldots$, estágio L. Um trabalho pode pular qualquer número de estágios contanto que ele seja processado em pelo menos um dos estágios;

- Cada trabalho $\mathrm{j}$ requer o tempo de processamento pjk no estágio $\mathrm{k}$.

Além disso, segundo Li et al. (2018) todos as tarefas e máquinas devem estar disponíveis no tempo zero, nenhuma tarefa pode ser interrompida durante a sua execução, cada máquina processa somente uma tarefa por vez e existe um buffer infinito entre dois estágios consecutivos. Um esquemático básico do HFS pode ser observado na Figura 1, nesse esquemático é representada uma produção com L estágios, M número de máquinas por estágio e um buffer entre os estágios.

Figura 1 - Esquema básico do HFS

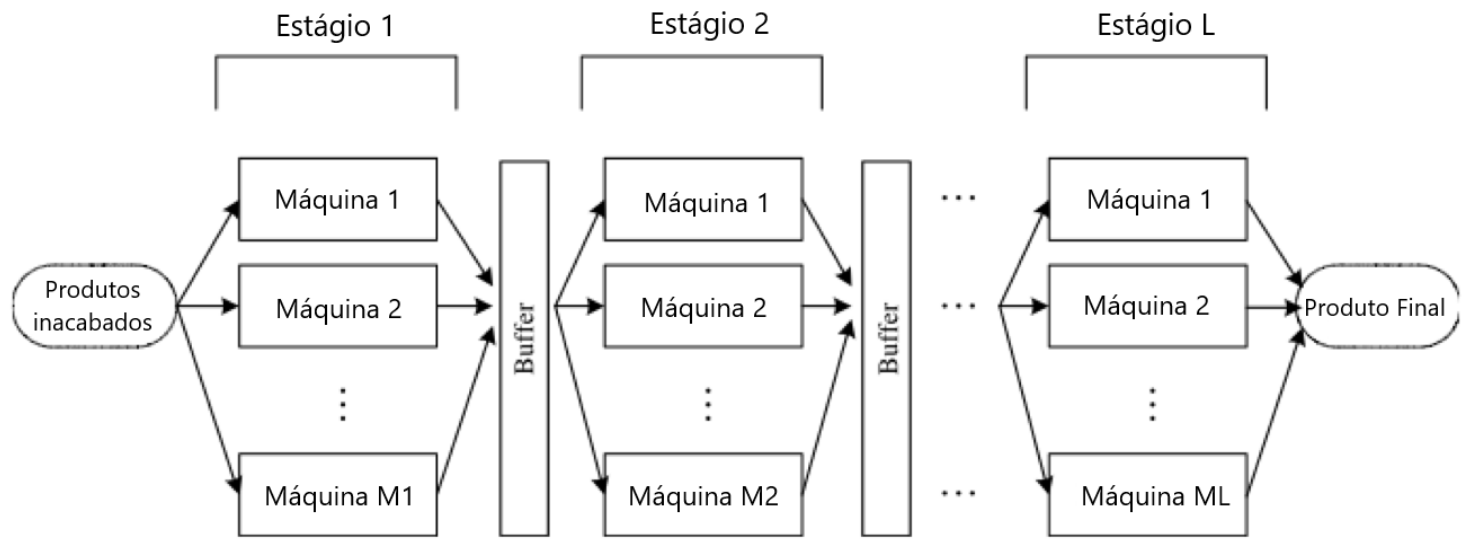

Fonte: Adaptado de Quadt e Kuhn (2007) 
As variações dos problemas HFS podem ser classificadas sob três aspectos. O primeiro aspecto é a configuração da produção que indica a quantidade de estágios, a quantidade de máquinas por estágio e as características das máquinas em cada estágio. O segundo aspecto são as restrições, que além das restrições do HFS servem para caracterizar o problema. O último aspecto é a função objetivo considerada, que pode ser desde parâmetros como o tempo total de produção até objetivos como o consumo de energia, a emissão de gases e outros.

\subsection{Formulação do problema}

Assim como no exemplo anterior nas instâncias avaliadas neste trabalho todas as tarefas e máquinas devem estar disponíveis no tempo zero, nenhuma tarefa pode ser interrompida durante a sua execução, cada máquina processa somente uma tarefa por vez e existe um buffer infinito entre dois estágios consecutivos. O desenvolvimento deste trabalho será dividido em duas partes, a primeiro adotando uma abordagem de único objetivo e a segunda multiobjetivo.

\subsection{1. Único objetivo}

O critério utilizado para avaliação dos resultados é o tempo total de produção (makespan), deste modo a função objetivo é:

$\operatorname{minCmax}$

Sendo: Cmax = tempo total de produção.

\subsubsection{Multiobjetivo}

A busca multiobjetivo otimizará o problema levando em conta o tempo total de produção (makespan) e o consumo de energia elétrica, conforme observado a seguir:

$\min \mathrm{f} 1(\mathrm{x}) ; \mathrm{f} 2(\mathrm{x})$

$\mathrm{f} 1(\mathrm{x})=\min C \max$

$\mathrm{f} 2(\mathrm{x})=$ minEtotal

sendo:

Cmax $=$ tempo total de produção.

Etotal $=$ consumo de energia elétrica total . 


\section{Revisão de literatura}

A revisão de literatura se deu a partir dos termos Optmization, Energy Consumption, Hybrid Flow Shop, Flexible flow shop, flow shop with parallel machines, Scheduling e Genetic Algorithm. A busca foi realizada primariamente com o auxílio do portal de periódicos capes com a assistência da ferramenta de organização bibliográfica mendeley. Os termos Hybrid Flow Shop, Flexible flow shop e flow shop with parallel machines são sinônimos (RIBAS; LEISTEN; FRAMIÑAN, 2010)(QUADT; KUHN, 2007),

Vários estudos de problemas de programação da produção híbrida propuseram diferentes métodos para a otimização do consumo de energia elétrica, a seguir são destacados alguns trabalhos importantes relacionados ao tema.

Lu et al. (2017) propôs um algoritmo híbrido multiobjetivo de busca com retrocesso com o objetivo de reduzir o consumo de energia e o tempo total de produção. $\mathrm{O}$ autor faz a comparação dos seus resultados com os algoritmos NSGA-II e MOEA/D.

Mokhtari e Hasani (2017) propôs um algoritmo híbrido entre o recozimento simulado e o algoritmo genético, o autor considerou 3 funções objetivo, a minimização do tempo total de produção, a maximização da disponibilidade do sistema e a minimização dos custos com energia da produção e da manutenção. Os resultados foram comparados estatisticamente com o recozimento simulado e o algoritmo genético.

Piroozfard, Wong e Wong (2018) propôs um algoritmo genético multiobjetivo, MOGA, com o objetivo de minimizar o atraso dos trabalhos, e a pegada de carbono, calculada de forma indireta pelo consumo de energia elétrica. Os resultados foram comparados com os 18 algoritmos NSGA-II, SPEA2.

Keller, Schönborn e Reinhart (2015) utilizou recozimento simulado para otimizar o consumo de energia, e faz a comparação com os resultados do algoritmo de inicialização e programação regressiva, o autor alegou uma redução nos custos com energia de 42,1\%.

Zhang e Chiong (2016) propôs um algoritmo genético multiobjetivo com busca local aprimorada, com o objetivo de minimizar o atraso ponderado médio e a energia total consumida. Os resultados foram comparados com o algoritmo NSGA-II, de forma a melhorar 
a comparação o autor integrou um procedimento de busca local iterativa para melhorar os resultados do NSGA-II.

Ding, Song e Wu (2016) propôs dois algoritmos multiobjetivo, MONEH e MMOIG, com o objetivo de minimizar o atraso ponderado médio e energia consumida total. Os resultados foram comparados com o algoritmo NSGA-II. Liu et al. (2016) propôs um algoritmo genético multiobjetivo, com o objetivo de minimizar o atraso dos trabalhos, e o consumo total de energia elétrica nos períodos de não processamento. Os resultados foram comparados com os algoritmos NSGA-II, SPEA2.

Li et al. (2018) propôs um algoritmo de otimização multiobjetivo considerando o tempo total de produção e o consumo de energia. O autor comparou os resultados com NSGA-II, MOEA/D DBEA e EADD.

Luo et al. (2013) propôs uma nova otimização de colônia de formigas considerando o tempo total de produção e o consumo de energia total. Os resultados foram comparados com os algoritmos NSGA-II e SPEA2.

Mansouri, Aktas e Besikci (2016) propôs uma otimização linear multiobjetivo e considerou as funções objetivo: tempo total de produção e a energia consumida total. $\mathrm{O}$ autor comparou o impacto de considerar a energia consumida total no tempo total de produção, além disso o autor validou os resultados do seu algoritmo com o software CPLEX.

Yan et al. (2016) propôs um algoritmo genético, com o objetivo de minimizar o tempo total de produção e o consumo de energia total. Os resultados foram comparados com dados da indústria.

\section{Metodologia}

A metodologia empregada nesse trabalho consiste em 8 etapas, conforme descrição do quadro 1. As etapas são descritas, em detalhes, nos parágrafos seguintes. 
Quadro 1 - Esquema básico do HFS

\begin{tabular}{cc}
\hline Etapa & Título \\
\hline 1 & Codificar as bases de dados. \\
2 & Definir o modelo de codificação da solução no algoritmo \\
genético. \\
3 & Definir as estratégias de seleção, cruzamento e mutação. \\
4 & Definir a estratégia de busca local \\
5 & Avaliar os resultados. \\
6 & Criar uma base de dados incluindo valores de consumo \\
7 & de energia. \\
8 & Codificar o algoritmo genético para uma estratégia multiobjetivo. \\
\hline
\end{tabular}

Fonte: Elaborado pelo autor, 2020.

Para avaliar o desempenho do algoritmo desenvolvido é necessário codificar uma base de dados, de forma a padronizar a entrada de dados no algoritmo. A codificação de entrada foi baseada em Mastrolilli (2000). A codificação da solução do problema foi utilizado o método descrito em Li e Gao (2016), essa codificação divide a solução em dois vetores, OS e MS, sendo o primeiro um método de representação baseado nas operações e o segundo representando as maquinas selecionadas para as operações de cada trabalho.

O vetor OS representa a ordem em que as operações devem ser executadas através de uma permutação dos números dos trabalhos. Como no problema abordado nesse trabalho as operações devem ser executadas em uma sequência determinada, assim as operações são representadas com os índices dos seus trabalhos correspondentes. Lendo os dados do vetor OS, da esquerda para a direita, cada vez que o índice de um trabalho aparece representa as suas operações de forma ordenada. O tamanho do vetor OS é igual número total de operações de todos os trabalhos.

O vetor MS indica a seleção das máquinas para as operações, nesse vetor as suas posições, da esquerda para a direita, representam as operações ordenadas seguindo a sequência dos índices dos trabalhos, essa sequência não difere de uma solução para outra, assim a máquina selecionada para cada operação é definida pelo número da máquina em cada posição do vetor.

No desenvolvimento do algoritmo genético podem ser adotadas diversas estratégias de seleção, cruzamento e mutação. A estratégia de seleção adotada foi a combinação da seleção por torneio e a seleção elitista, parte da população é selecionada por elitismo, ou seja são 
selecionados os melhores indivíduos determinados pela função objetivo, e o restante da população é selecionado por torneio, onde um grupo maior ou igual a 2 de indivíduos são selecionados aleatoriamente e o indivíduo mais apto é selecionado. Nesse trabalho $10 \%$ dos indivíduos foram selecionados por elitismo e o restante por sorteio, a cada seleção são escolhidos 30 indivíduos, mesmo número da população inicial. A estratégia de cruzamento consiste na combinação de um cruzamento de dois pontos, a probabilidade de cruzamento adotada nesse trabalho foi de $80 \%$. A estratégia de mutação consiste na troca da posição de operações e da seleção de máquinas de forma aleatória com uma probabilidade $15 \%$.

Segundo Blum e Roli (2003) é muito importante o equilíbrio entre diversificação e intensificação, o algoritmo genético é uma excelente metaheurística para diversificação, desse modo foi utilizado uma estratégia de busca local baseada em Mastrolilli e Gambardella (2000). A estratégia adotada consiste em selecionar um indivíduo aleatoriamente e fazer uma busca local na vizinhança selecionando o indivíduo mais apto.

As instâncias instancias publicadas por Hurink, Jurisch e Thole (1994) são utilizadas para avaliar os resultados do algoritmo e ajustar os seus parâmetros. Então foram geradas 20 novas instâncias, incluindo valores aleatórios para o consumo de energia elétrica, atendendo ao seguintes requisitos:

- O número de trabalhos pode assumir qualquer valor inteiro entre 3 e 5 ;

- O número de máquinas pode assumir qualquer valor inteiro entre 2 e 6;

- O tempo de processamento pode assumir qualquer valor inteiro entre 2 e 8 ;

- O consumo de energia elétrica pode assumir qualquer valor inteiro entre 1 e 3 ;

Um novo algoritmo foi codificado para incluir na função objetivo o valor da energia elétrica, este novo objetivo foi incorporado na seleção por torneio, mantendo-se na seleção por elitismo somente o critério do tempo total de produção. Na seleção por torneio $50 \%$ dos indivíduos foram selecionados por sua aptidão ao tempo total de produção e $50 \%$ ao consumo de energia elétrica.

Por fim foi avaliado os impactos no tempo total de produção da incorporação do objetivo de reduzir o consumo de energia elétrica. 


\section{Resultados e discussão}

O algoritmo gerado nesse trabalho foi comparado com instâncias de referência da literatura. Foram avaliadas 9 instâncias de Hurink, Jurisch e Thole (1994), conforme observado na Tabela 1. A coluna LB (lower bound) apresenta os melhores valores da literatura, observados em Li e Gao (2016b). A coluna melhor resultado apresenta os melhores valores alcançados neste trabalho, a coluna média dos resultados apresenta a média dos resultados alcançados nesse trabalho em 10 repetições, a coluna desvio padrão dos resultados apresenta o desvio padrão das 10 repetições e a coluna gap apresenta a diferença percentual entre o menor valor da literatura e o melhor resultado alcançado neste trabalho. Pode-se observar que os gaps foram inferiores a $4 \%$ e os melhores resultados da literatura foram alcançados em $55 \%$ das instâncias avaliadas.

Tabela 1 - Resultados, objetivo: tempo total de produção.

\begin{tabular}{cccccc}
\hline Instância & LB & $\begin{array}{c}\text { Melhor } \\
\text { resultado }\end{array}$ & $\begin{array}{c}\text { Média dos } \\
\text { resultados }\end{array}$ & $\begin{array}{c}\text { Desvio } \\
\text { padrão dos } \\
\text { resultados }\end{array}$ & GAP \\
\hline la01 & 609 & 609 & 622,8 & 8,9 & $0 \%$ \\
la02 & 655 & 655 & 681,0 & 17,6 & $0 \%$ \\
la03 & 550 & 561 & 570,7 & 8,3 & $2 \%$ \\
la04 & 568 & 591 & 600,5 & 7,6 & $4 \%$ \\
la05 & 503 & 503 & 512,7 & 0,0 & $0 \%$ \\
la06 & 833 & 833 & 838,9 & 7,5 & $0 \%$ \\
la07 & 762 & 791 & 802,3 & 9,7 & $4 \%$ \\
la08 & 845 & 845 & 853,5 & 8,7 & $0 \%$ \\
la09 & 878 & 886 & 900,6 & 9,5 & $1 \%$ \\
\hline
\end{tabular}

Fonte: Elaborado pelo autor, 2020.

A Tabela 1 demostra a capacidade do algoritmo desenvolvido neste trabalho de obter bons resultados, dessa forma o algoritmo desenvolvido neste trabalho foi avaliado nas instancias geradas. As instâncias foram avaliadas de forma a reduzir somente o tempo total de produção e com duplo objetivo de reduzir o tempo total de produção e o consumo de energia elétrica. As instâncias ck01 a ck20 foram avaliadas 5 vezes cada, para cada objetivo, e os valores de tempo total de produção foram os mesmos. A Tabela 2 mostra os resultados com o objetivo de reduzir o tempo total de produção, a Tabela 3 mostra os resultados com o objetivo de reduzir o tempo total de produção e o consumo de energia. 
Tabela 2 - Resultados, objetivo: tempo total de produção.

\begin{tabular}{cccc}
\hline Instância & $\begin{array}{c}\text { Média do tempo } \\
\text { total de produção }\end{array}$ & $\begin{array}{c}\text { Média Consumo de } \\
\text { energia elétrica }\end{array}$ & $\begin{array}{c}\text { Desvio padrão do } \\
\text { Consumo de } \\
\text { energia elétrica }\end{array}$ \\
\hline ck01 & 15 & 216,8 & 14,3 \\
ck02 & 36 & 257,6 & 12,8 \\
ck03 & 19 & 236,0 & 13,5 \\
ck04 & 23 & 111,0 & 0,0 \\
ck05 & 18 & 120,6 & 14,0 \\
ck06 & 15 & 125,6 & 10,7 \\
ck07 & 16 & 167,8 & 22,0 \\
ck08 & 17 & 160,6 & 9,9 \\
ck09 & 23 & 177,8 & 3,0 \\
ck10 & 20 & 156,2 & 7,7 \\
ck11 & 20 & 136,2 & 12,3 \\
ck12 & 16 & 169,0 & 29,1 \\
ck13 & 18 & 114,2 & 6,9 \\
ck14 & 14 & 92,6 & 1,3 \\
ck15 & 21 & 236,2 & 22,3 \\
ck16 & 13 & 47,6 & 2,6 \\
ck17 & 19 & 218,8 & 12,3 \\
ck18 & 18 & 178,6 & 14,8 \\
ck19 & 19 & 117,2 & 20,2 \\
ck20 & 14 & 98,6 & 1,3 \\
\hline
\end{tabular}

Fonte: Elaborado pelo autor, 2020.

As diferenças percentuais observadas na Tabela 4 demonstram o potencial de redução do consumo de energia deste trabalho sem que seja impactado o tempo de produção. É possível verificar na segunda coluna que o tempo total de produção não foi afetado em nenhum dos casos, entretanto foi possível observar uma redução no consumo de energia de até $26 \%$. 
Tabela 3 - Resultados, objetivo: tempo total de produção e consumo de energia elétrica.

\begin{tabular}{lccc}
\hline Instância & $\begin{array}{c}\text { Média do tempo } \\
\text { total de produção }\end{array}$ & $\begin{array}{c}\text { Média Consumo de } \\
\text { energia elétrica }\end{array}$ & $\begin{array}{c}\text { Desvio padrão do } \\
\text { Consumo de } \\
\text { energia elétrica }\end{array}$ \\
\hline ck01 & 15 & 203,6 & 16,3 \\
ck02 & 36 & 242,0 & 12,4 \\
ck03 & 19 & 211,2 & 20,3 \\
ck04 & 23 & 111,0 & 0,0 \\
ck05 & 18 & 106,6 & 8,1 \\
ck06 & 15 & 119,6 & 5,4 \\
ck07 & 16 & 145,2 & 14,2 \\
ck08 & 17 & 156,0 & 2,7 \\
ck09 & 23 & 174,4 & 1,3 \\
ck10 & 20 & 146,2 & 9,4 \\
ck11 & 20 & 122,2 & 1,1 \\
ck12 & 16 & 125,8 & 12,8 \\
ck13 & 18 & 90,0 & 7,0 \\
ck14 & 14 & 92,0 & 2,1 \\
ck15 & 21 & 178,2 & 20,3 \\
ck16 & 13 & 48,4 & 2,0 \\
ck17 & 19 & 218,8 & 6,4 \\
ck18 & 18 & 172,0 & 0,0 \\
ck19 & 19 & 103,6 & 12,9 \\
ck20 & 14 & 97,8 & 4,5 \\
\hline & & & \\
\hline
\end{tabular}

Fonte: Elaborado pelo autor, 2020. 
Tabela 4 - Aumentos percentuais ao considerar o consumo de energia elétrica.

\begin{tabular}{ccc}
\hline Instância & $\begin{array}{c}\text { Aumento } \\
\text { percentual do } \\
\text { tempo total de } \\
\text { produção }\end{array}$ & $\begin{array}{c}\text { Aumento } \\
\text { percentual do } \\
\text { Consumo de } \\
\text { energia elétrica }\end{array}$ \\
\hline ck01 & $0,0 \%$ & $-6,0 \%$ \\
ck02 & $0,0 \%$ & $-6,0 \%$ \\
ck03 & $0,0 \%$ & $-11,0 \%$ \\
ck04 & $0,0 \%$ & $0,0 \%$ \\
ck05 & $0,0 \%$ & $-12,0 \%$ \\
ck06 & $0,0 \%$ & $-5,0 \%$ \\
ck07 & $0,0 \%$ & $-13,0 \%$ \\
ck08 & $0,0 \%$ & $-3,0 \%$ \\
ck09 & $0,0 \%$ & $-2,0 \%$ \\
ck10 & $0,0 \%$ & $-6,0 \%$ \\
ck11 & $0,0 \%$ & $-10,0 \%$ \\
ck12 & $0,0 \%$ & $-26,0 \%$ \\
ck13 & $0,0 \%$ & $-21,0 \%$ \\
ck14 & $0,0 \%$ & $-1,0 \%$ \\
ck15 & $0,0 \%$ & $-25,0 \%$ \\
ck16 & $0,0 \%$ & $2,0 \%$ \\
ck17 & $0,0 \%$ & $0,0 \%$ \\
ck18 & $0,0 \%$ & $-4,0 \%$ \\
ck19 & $0,0 \%$ & $-12,0 \%$ \\
ck20 & $0,0 \%$ & $-1,0 \%$ \\
\hline
\end{tabular}

Fonte: Elaborado pelo autor, 2020.

\section{Conclusões}

Nesse artigo um algoritmo genético foi proposto para o problema da programação da produção híbrida com o objetivo de reduzir o consumo de energia elétrica no ambiente produtivo, 9 instâncias da literatura foram utilizadas para testar o desempenho do algoritmo e foram geradas 20 instâncias de forma aleatória que incluíam além do número de trabalhos, quantidade de máquinas e tempos de processamento os consumos de energia elétrica de cada máquina. As instâncias geradas foram testadas com o objetivo de reduzir o tempo total de produção e com o duplo objetivo de reduzir o consumo de energia elétrica e o tempo total de produção.

Os resultados desse trabalho demonstraram o potencial de redução do consumo de energia elétrica decorrente do uso de técnicas de programação da produção que não levem somente 
em conta parâmetros produtivos como o tempo total de produção, mas também considerem outros objetivos como a redução do consumo de energia.

No futuro, os seguintes trabalhos podem ser realizados. Primeiro, a expansão das instâncias da literatura utilizadas para testar o desempenho do algoritmo e segundo, a geração de um maior número de instâncias, incluindo instâncias de maior porte e faixas diferentes para os tempos de produção e os consumos de energia.

\section{REFERÊNCIAS}

BLUM, C.; ROLI, A. Metaheuristics in combinatorial optimization: Overview and conceptual comparison. ACM computing surveys (CSUR), Acm, v. 35, n. 3, p. 268-308, 2003.

CORMEN, T. H. et al. Introduction to algorithms. [S.1.]: MIT press, 2009.

DING, J.-Y.; SONG, S.; WU, C. Carbon-efficient scheduling of flow shops by multi-objective optimization. European Journal of Operational Research, v. 248, n. 3, p. 758-771, 2016.

EPE. Anuário estatístico de Energia Elétrica 2017. Brasília: [s.n.], 2017.

FAN, K. et al. Review and classification of hybrid shop scheduling. Production Engineering, v. 12, n. 5, p. 597-609, 2018.

GUPTA, J. N. D. Two-Stage, Hybrid Flowshop Scheduling Problem. Journal of the Operational Research Society, v. 39, n. 4, p. 359, 1988.

HURINK, J.; JURISCH, B.; THOLE, M. Tabu search for the job-shop scheduling problem with multi-purpose machines. Operations-Research-Spektrum, v. 15, n. 4, p. 205-215, Dec 1994.

KELLER, F.; SCHÖNBORN, C.; REINHART, G. Energy-orientated Machine Scheduling for Hybrid Flow Shops. Procedia CIRP, v. 29, p. 156-161, 2015.

LI, J.-q. et al. Efficient multi-objective optimization algorithm for hybrid flow shop scheduling problems with setup energy consumptions. Journal of Cleaner Production, v. 181, p. 584-598, 2018.

LI, X.; GAO, L. An effective hybrid genetic algorithm and tabu search for flexible job shop scheduling problem. International Journal of Production Economics, v. 174, p. 93-110, 2016.

LIU, Y. et al. A multi-objective genetic algorithm for optimisation of energy consumption and shop floor production performance. International Journal of Production Economics, v. 179, p. 259-272, 2016.

LU, C. et al. Energy-efficient permutation flow shop scheduling problem using a hybrid multi-objective backtracking search algorithm. Journal of Cleaner Production, v. 144, p.228-238, 2017.

LUO, H. et al. Hybrid flow shop scheduling considering machine electricity consumption cost. International Journal of Production Economics, Elsevier, v. 146, n. 2, p. 423-439, dec 2013.

MANSOURI, S. A.; AKTAS, E.; BESIKCI, U. Green scheduling of a two-machine flowshop: Trade-off etween makespan and energy consumption. European Journal of Operational Research, v. 248, n. 3, p. 772-788, 2016.

MASTROLILLI, M.; GAMBARDELLA, L. M. Effective neighbourhood functions for the flexible job shop problem. Journal of scheduling, Wiley Online Library, v. 3, n. 1, p. 3-20, 2000. 
MOKHTARI, H.; HASANI, A. An energy-efficient multi-objective optimization for flexible job-shop scheduling problem. Computers and Chemical Engineering, v. 104, p. 339-352, 2017.

PEZZELLA, F.; MORGANTI, G.; CIASCHETTI, G. A genetic algorithm for the Flexible Job-shop Scheduling Problem. Computers \& Operations Research, v. 35, n. 10, p. 3202-3212, 2008.

PINEDO, M. L. Scheduling. 5aedição. ed. Cham: Springer International Publishing, 2016. ISBN 978-3-31926578-0.

PIROOZFARD, H.; WONG, K. Y.; WONG, W. P. Minimizing total carbon footprint and total late work criterion in flexible job shop scheduling by using an improved multi-objective genetic algorithm. Resources, Conservation \& Recycling, v. 128, p. 267-283, 2018.

QUADT, D.; KUHN, H. A taxonomy of flexible flow line scheduling procedures. European Journal of Operational Research, v. 178, n. 3, p. 686-698, may 2007.

RIBAS, I.; LEISTEN, R.; FRAMIÑAN, J. M. Review and classification of hybrid flow shop scheduling problems from a production system and a solutions procedure perspective. Computers \& Operations Research, Pergamon, v. 37, n. 8, p. 1439-1454, aug 2010.

RUIZ, R.; VÁZQUEZ-RODRÍGUEZ, J. A. The hybrid flow shop scheduling problem. European Journal of Operational Research, North-Holland, v. 205, n. 1, p. 1-18, aug 2010.

WORLDBANK. Value Added (\% of GDP). 2016. Disponível em: $\langle$ https://data.worldbank.org/indicator/NV.IND.MANF.ZS〉.

YAN, J. et al. A multi-level optimization approach for energy-efficient flexible flow shop scheduling. Journal of Cleaner Production, v. 137, p. 1543-1552, 2016.

ZHANG, R.; CHIONG, R. Solving the energy-efficient job shop scheduling problem: a multi-objective genetic algorithm with enhanced local search for minimizing the total weighted tardiness and total energy consumption. Journal of Cleaner Production, v. 112, p. 3361-3375, 2016. 